\title{
PARABOLIC BURSTING, SPIKE-ADDING, DIPS AND SLICES IN A MINIMAL MODEL*
}

\author{
Mathieu Desroches ${ }^{1,2, * *}$, JeAn-Pierre Francoise ${ }^{3}$ \\ AND MARTIN KRUPA ${ }^{1,2,4}$
}

\begin{abstract}
A minimal system for parabolic bursting, whose associated slow flow is integrable, is presented and studied both from the viewpoint of bifurcation theory of slow-fast systems, of the qualitative analysis of its phase portrait and of numerical simulations. We focus the analysis on the spike-adding phenomenon. After a reduction to a periodically forced one-dimensional system, we uncover the link with the dips and slices first discussed by J.E. Littlewood in his famous articles on the periodically forced van der Pol system.
\end{abstract}

Mathematics Subject Classification. 37C10, 37C27, 34C23, 37G15, 34C15, 34E17.

Received October 26, 2018. Accepted April 24, 2019.

\section{INTRODUCTION}

Bursting is an essential rhythm of neural activity, whether it be intrinsic or extrinsic (upon the application of an external stimulus); ubiquitous in biological data, in particular from the brain, it can be modelled with mathematical equations within different frameworks, in particular parametrised ordinary differential equations (ODEs) with multiple timescale. Slow-fast models of bursting activity have been developed, both biophysically and phenomenologically, and analysed since the 1970s. The seminal work of Rinzel in the mid-1980s [39, 40] has lead to a first classification of bursting patterns using mathematical criteria, in particular bifurcation theory. Later on, this first classification was completed by Izhikevich [26] generalising the idea of using bifurcations of an auxiliary so-called fast subsystem to initiate and terminate the burst of a bursting system. Further bursting classification works were obtained in $[6,19]$. The third class of Rinzel is denoted parabolic bursting and it corresponds to a SNIC-SNIC bursting in the taxonomy introduced by Izhikevich, SNIC standing for saddle-node (bifurcation) on an invariant circle. This is because the underlying slow-fast bursting system has a two-dimensional fast subsystem displaying a two-parameter family of SNIC bifurcations, which organises the transition from quiescence to burst and back in the full system. The main result of the present paper is the

* The first two authors are quite gratefull to the ANR "ISDEEC" for a financial support.

Keywords and phrases: Parabolic bursting, spike-adding, Littlewood's dips and slices.

1 MathNeuro Team, Inria Sophia Antipolis - Méditerranée, Sophia Antipolis, France.

2 Université Côte d'Azur, Nice, France.

3 Laboratoire Jacques-Louis Lions, Sorbonne Université, Paris, France.

${ }^{4}$ Université de Nice Sophia Antipolis, Laboratoire J.-A. Dieudonné, Nice, France.

** Corresponding author: mathieu.desroches@inria.fr 
(a)

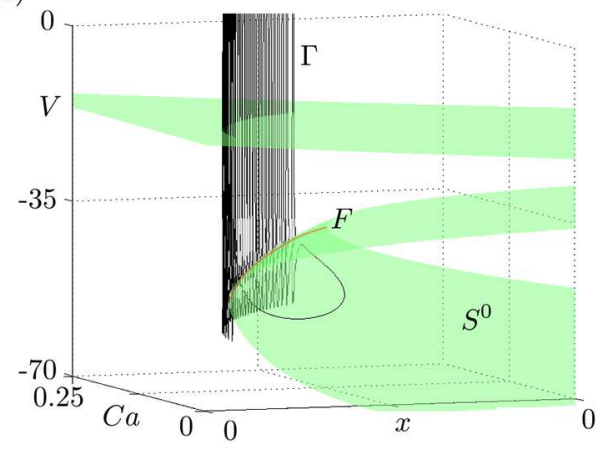

(c)

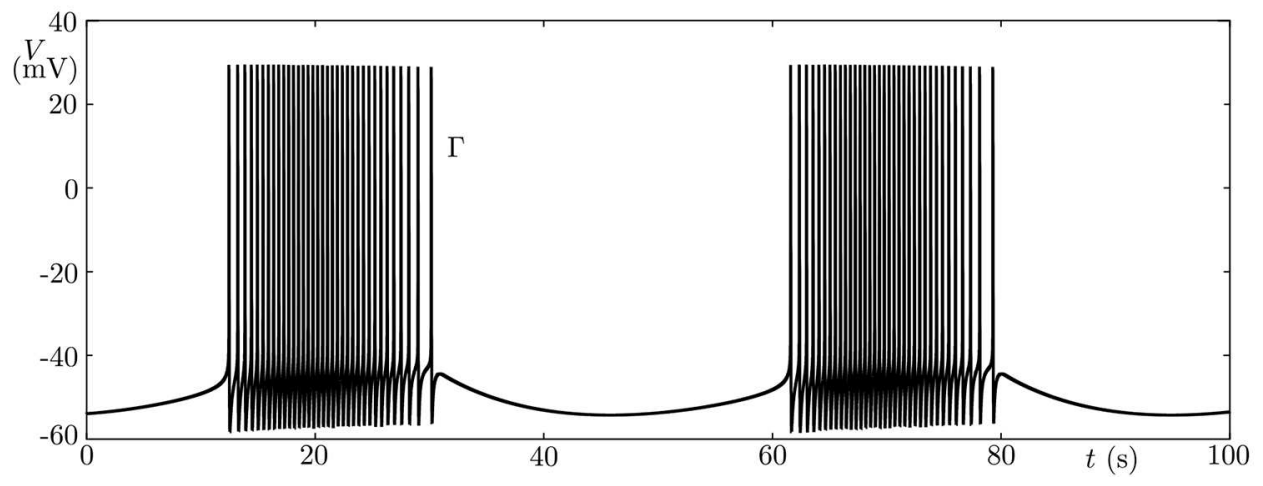

(b)

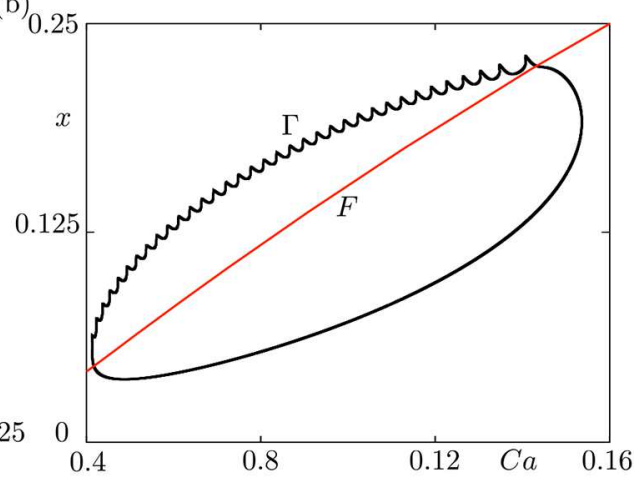

Figure 1. Parabolic bursting in Plant model for the R15 neuron of the Aplysia.

introduction of a new parabolic burster, whose associated slow subsystem is integrable and hence in which the spike-adding is more easily analysed. A new light is shed onto this spike-adding structure that organises the burst size in this class of bursting system. Our system has a cylindrical critical manifold with two fold lines along each of which one can find conditions for so-called folded-saddle singularities and associated canard solutions to exist; recall that canards are solutions staying close to repelling manifolds of slow motion for a long time interval $[2,17,30]$. Depending on the slow periodic forcing, we obtain spike-adding canard explosions [12, 47] organising the transition from slow oscillation to bursting by passing near one or both folded saddles. In every case, the scenario is the same: the first spike is added via a slow passage near the folded saddle and its associated canard, whereas subsequent ones are added via passages near a jump-on point [11] (a point on the fold line where the slow flow points away from the fold in both directions) and a repulsive segment of the folded-saddle canard solution. This provides one step further in understanding better the salient features of parabolic bursting in order to improve current models.

The first important reference on the observation of parabolic bursting in biological data is the famous Plant model on the R15 neuron of the Aplysia, which is a mollusk [35-38]. This model is constructed within the conductance framework introduced by Nobel prize winners Alan Hodgkin and Andrew Huxley in their seminal 1952 paper [25]. This five-dimensional model is not singularly perturbed as it does not have a explicit small parameter, however it clearly displays slow-fast dynamics, in the form of parabolic bursting limit cycles, and one can identify 3 fast variables and 2 slow variables. The main state variable is the membrane potential $V$ and it is fast; the two slow variables, $x$ and $C a$, are both related to calcium. A typical parabolic bursting cycle $\Gamma$ produced by this model is shown in Figure 1 . Figure 1a shows a phase-space projection onto the $(x, C a, V)$-space, where the green surface is the fast nullsurface obtained by solving for $V$ the (implicit) equilibrium equation for 
the fast variables over a range of values of the slow variables $x$ and $C a$; such a fast nullsurface is referred to as the critical manifold $S^{0}$ of the system. The slow dynamics occurs very close to the lower attracting sheets of $S^{0}$ and the transition to the fast dynamics occurs as the trajectory reaches the fold curve $F$ of $S^{0}$. In the particular case of parabolic bursting, the transitions from the quiescent (slow) phase to the burst and back both correspond to a passage near $F$, which is a curve of SNIC bifurcation points of the fast subsystem; this justifies that the burst starts and terminates with very low frequency. This is better observed when further projecting the orbit together with the SNIC curve $F$ onto the plane of slow variables; this is done in Figure $1 \mathrm{~b}$ where one can clearly observe the two phases of such a parabolic bursting cycle, separated by $F$, the slow phase to the right of $F$ and the fast phase or burst to the left. Figure 1c shows the membrane potential time profile for the bursting cycle $\Gamma$. This highlights the typical features of parabolic bursting. Namely, first the fact that this bursting scenario can be seen as recurrent groups of spikes (bursts) on top of a slow oscillation that is possible due to the presence of two slow variables, here $x$ and $C a$; second, the fact that the interspike interval during each burst is large at the beginning and at the end of the burst, hence giving a parabolic-shaped curve if plotted against time. The number of spikes per burst depends very nonlinearly on key parameters of the model (e.g. the sodium maximal conductance) and, from slow spikeless cycles in some parameter regime, the solutions can gain more and more spikes as a parameter is varied. These spike-adding transitions were recently analysed in the framework of geometric singular perturbation theory in [13] and it was shown that they are organised by canards of folded-saddle type; see also [14].

There are many other examples of parabolic bursting in biophysical models; see e.g. [1, 10, 29, 44]. We like to mention recent contributions to the electrical activity of GnRH neurones. In [34], Moenter et al. model the fact that GnRH secreting neurons may exhibit parabolic bursting patterns of activity, where the burst is superimposed onto a slow wave representing a rhythm linked to secretory activity; for other type of secretory activity in GnRH neuron models, see [9]. Minimal phase models of parabolic bursters were first introduced by Ermentrout and Kopell with the so-called theta model [18], sometimes also referred to as Atoll model [28]; see also [45]. Spike-adding via canards in the Atoll model was related to jump-on points in [13]. More generally, canards in slow-fast planar systems defined on the two-torus were studied by Guckenheimer and Ilyashenko in [24] and then by Schurov and collaborators in [41-43] however without any link made to parabolic bursting or spike-adding.

In this article, we present a new minimal model for parabolic bursting (in Sect. 2) which displays an integrable associated slow subsystem; note that a versal deformation of the SNIC needs precisely two parameters. We analyse this minimal system by putting the emphasis on the spike-adding scenario which distinguishes the first transition where spike addition is based on folded-saddle canards, whereas the subsequent ones are added through jump-on points (still near a folded saddle). We also describe the folded homoclinic bifurcation that takes places in the slow subsystem and which was already identified in [13] as a key ingredient for spike adding in parabolic bursters. We then show a progressive reduction of the initial model to a one-dimensional ODE with periodic forcing allowing comparison with the forced van der Pol system. This leads us (in Sect. 3) to the equivalence between headless canards and dips, on the one hand, and canards with head and slices, on the other hand. These special solutions were introduced by Littlewood in one article of his seminal series on the forced van der Pol and it provides essentially a first historical encounter with canard solutions. We describe in detail the geometry of dips and slices in time series where the underlying (time-dependent) fast nullcline of the system can be represented and helps to shed light on the slow-fast effects underpinning canard dynamics.

\section{A MINIMAL MODEL}

We now consider a minimal SNIC oscillator, namely

$$
\begin{aligned}
& \dot{r}=r\left(1-r^{2}\right), \\
& \dot{\theta}=a-\sin \theta,
\end{aligned}
$$


or written in cartesian coordinates

$$
\begin{aligned}
& \dot{x}=x\left(1-x^{2}-y^{2}\right)-y\left(a-\frac{y}{\sqrt{x^{2}+y^{2}}}\right), \\
& \dot{y}=y\left(1-x^{2}-y^{2}\right)+x\left(a-\frac{y}{\sqrt{x^{2}+y^{2}}}\right) .
\end{aligned}
$$

We then force this minimal SNIC oscillator periodically in $a$ through the following slow harmonic oscillator

$$
\begin{aligned}
a^{\prime} & =\varepsilon\left(\mu-\mu_{c}\right), \\
\mu^{\prime} & =\varepsilon\left(a_{c}-a\right),
\end{aligned}
$$

where $\mu_{c}$ and $a_{c}$ are two real parameters that determine the centre of all periodic orbits solutions to system (2.3).

Gathering systems (2.1) and (2.3) and reparametrising time gives the following two slow/two fast system expressed in the slow time

$$
\begin{aligned}
\varepsilon \dot{r} & =r\left(1-r^{2}\right), \\
\varepsilon \dot{\theta} & =a-\sin \theta, \\
\dot{a} & =\mu-\mu_{c}, \\
\dot{\mu} & =a_{c}-a .
\end{aligned}
$$

System (2.4) is a parabolic burster. Its cartesian critical manifold is the cylinder of equation

$$
S^{0}:=\left\{(x, y, a, \mu) \in \mathbb{R}^{4} \mid x^{2}+y^{2}=1, a=y\right\} .
$$

In polar coordinates, the critical manifold $S^{0}$ takes the form

$$
S^{0}=\{r=1, a=\sin \theta\}
$$

The two fold lines are given by the condition $\cos \theta=0$, that is, $\theta=\frac{\pi}{2} \bmod \pi$.

\subsection{Slow flow and desingularisation}

Taking the $\varepsilon=0$ limit of system (2.4) yields the so-called slow subsystem, also referred to as the reduced system (RS), which captures an approximation of the slow dynamics of the original system, constrained to evolve on the critical manifold $S^{0}$. In the present case, we get

$$
\begin{aligned}
& 0=r\left(1-r^{2}\right), \\
& 0=a-\sin \theta, \\
& \dot{a}=\mu-\mu_{c}, \\
& \dot{\mu}=a_{c}-a .
\end{aligned}
$$

System (2.7) is a differential-algebraic equation (DAE). One can gain information about the dynamics of the fast variables $r$ and $\theta$ in this singular limit, by differentiating the two algebraic equations of system (2.7) with respect to time. For the first one, this brings

$$
-2 r \dot{r}=0
$$


given the constraint of being on the critical manifold $S^{0}$. Equation (2.8) implies $\dot{r}=0$, which is consistent with the fact that $S^{0}$ is given by $\{r=1\}$. The second algebraic equation of $(2.7)$, once differentiated with respect to time, gives

$$
\dot{a}-\cos \theta \dot{\theta}=0,
$$

to which we add the two slow equations unaffected by the $\varepsilon=0$ limit in (2.7). As is customary since the critical manifold is two-dimensional, we project the resulting limiting system onto a phase plane which we choose to be the $(\theta, \mu)$-plane. We obtain the following reduced system

$$
\begin{aligned}
\cos \theta \dot{\theta} & =\mu-\mu_{c}, \\
\dot{\mu} & =a_{c}-\sin \theta,
\end{aligned}
$$

since we have $a=\sin \theta$ on $S^{0}$. System (2.10) is singular along the fold lines of $S^{0}$, that is, at points where $\cos \theta=0$. A classical tool to overcome this problem is to "extend" the reduced system to the fold lines by rescaling time by a factor $\cos \theta$. We obtain the so-called desingularised reduced system (DRS)

$$
\begin{aligned}
\theta^{\prime} & =\mu-\mu_{c}, \\
\mu^{\prime} & =\cos \theta\left(a_{c}-\sin \theta\right) .
\end{aligned}
$$

Equilibria of DRS systems located on the fold lines (which appear precisely through the time rescaling that transform the RS into the DRS) are the so-called pseudo-equilibria or folded singularities of the original system. Therefore one can conclude that system (2.4) has folded singularities at

$$
(\theta, \mu)=\left(\pi / 2 \bmod \pi, \mu_{c}\right) .
$$

The Jacobian matrix at $\left( \pm \frac{\pi}{2}, \mu_{c}\right)$ is given by

$$
\mathbf{J}^{ \pm}=\left(\begin{array}{cc}
0 & 1 \\
1 \mp a_{c} & 0
\end{array}\right)
$$

whose determinant is

$$
\operatorname{det}\left(\mathbf{J}^{ \pm}\right)=-1 \pm a_{c}
$$

Hence, for $\left|a_{c}\right|<1$, both folded singularities are of saddle type. The eigenvalues are:

$$
\lambda_{ \pm}^{ \pm}= \pm \sqrt{1 \mp a_{c}}
$$

and the eigendirections are defined by the linear relation

$$
\mu=\lambda \theta
$$

In the folded-saddle scenario, due to the $\theta$-dependent time rescaling defined above and which allows to resolve the singularities along the fold lines of the reduced system, the stable and unstable manifolds of the saddle equilibrium of the DRS correspond to special trajectories of the RS that cross the folded-saddle in finite time hence flowing along both the attracting and the repelling sheets of the critical manifold. One trajectory crosses at the folded saddle from the attracting side to the repelling one in forward time, and it corresponds to the stable manifold of the saddle of the DRS. Therefore it has a canard behaviour and it is called the true singular 
canard of the folded sadde. The other special trajectory instead crosses at the folded saddle from the repelling side to the attracting side in forward time and hence it has so-to-speak an anti-canard behaviour. It corresponds to the unstable manifold of the saddle equilibrium of the DRS and it is called the faux singular canard of the folded saddle. There is a degenerate case at $a_{c}=0$, where the spike-adding solutions visit both folded saddles in phase space; they only visit one of them (depending on the sign of $a_{c}$ ) as soon as $a_{c} \neq 0$. This is best understood by analysing the integrable structure of the DRS; see below and also Figures 2 and 3.

As showcased in [13], the presence of a folded saddle on the critical manifold of a parabolic burster organises the transition from the subthreshold (oscillatory) regime to the spiking and then the bursting regime, with the canard associated with the folded saddle organising spike-adding transitions. This type of dynamics also arises in three-time-scale systems as recently shown in [14]. An important object in this spike-adding scenario, unveiled in [13] and justified through qualitative arguments and numerical bifurcation analysis, is a so-called folded homoclinic bifurcation shown as blue and red curves in Figures 2 and 3; see also [14]. This object refers to a homoclinic bifurcation in the DRS which then, due to the time rescaling, corresponds in the RS to a limiting curve starting at the folded saddle and returning back to it with a finite passage time, hence connecting the faux canard and the true singular canards of the folded saddle. This object terminates the family of small limit cycles in the DRS. The particularity of the current DRS is that it is integrable, therefore one can find explicitly the folded homoclinic connection, as explained below.

\subsection{Folded homoclinic connection}

The DRS (2.11) is integrable with hamiltonian given by

$$
H(\theta, \mu)=-0.5 \mu^{2}+\mu_{c} \mu+a_{c} \sin \theta+0.25 \cos 2 \theta .
$$

Therefore, solutions of system (2.11) correspond to level sets of the function $H$, that is, the sets $\{H=h\}$ for constants $h$. Solving the corresponding algebraic equation with respect to $\mu$ gives the following characterisation

$$
\mu=\mu_{c} \pm \sqrt{\mu_{c}^{2}-\widetilde{h}}
$$

where $\widetilde{h}=2\left(h-a_{c} \sin \theta-0.25 \cos 2 \theta\right)$. Then, the homoclinic connection is the level set of $H$ that goes through each of the folded saddle $\left( \pm \frac{\pi}{2}, \mu_{c}\right)$, which yields $\widetilde{h}_{\text {hom }}^{ \pm}=\mu_{c}^{2}$, that is,

$$
h_{\mathrm{hom}}^{ \pm}=0.5 \mu_{c}^{2} \pm a_{c}-0.25 .
$$

Hence we obtain the equation for the folded homoclinic connections, namely

$$
\left\{(\theta, \mu) ;-0.5 \mu^{2}+\mu_{c}\left(\mu-0.5 \mu_{c}\right)+a_{c}(\sin \theta \mp 1)+0.25(\cos 2 \theta+1)=0\right\} .
$$

When $a_{c}=0$, there is no homoclinic connection in the DRS (2.11) but two double heteroclinic connections between the two folded saddles; indeed, equation (2.17) is clearly satisfied at $\left( \pm \frac{\pi}{2}, \mu_{c}\right)$ when $a_{c}=0$; see Figure 2. For $a_{c} \neq 0$, each folded saddle has a double homoclinic connection but only one of them interacts with canard solutions; see Figure 3.

\subsection{Canard-induced spike-adding transitions}

The spike-adding process in our minimal system (2.4) is mediated by canard solutions near each foldedsaddle singularity $\mathrm{fs}^{ \pm}$or both, depending on whether $a_{c}$ is nonzero or zero. When $a_{c}=0$, then solutions are symmetric with respect to $a$ and spikes are added two by two through transitions during which solutions come close to each folded saddle. Figure 2 presents this adding process for $a_{c}=0$. All central panels display solutions of system (2.4) for different initial conditions, together with the folded homoclinic connection and the folded 
(a1)

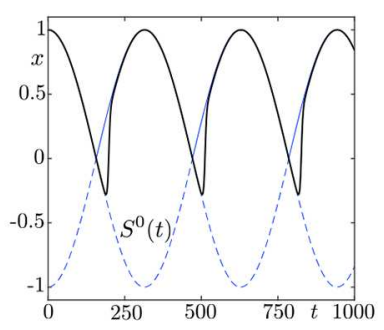

(a2)

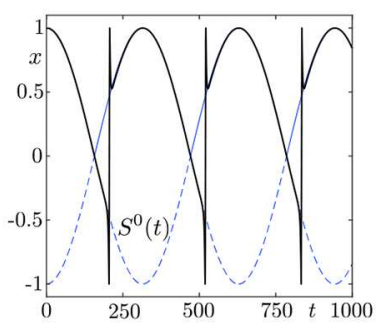

(a3)

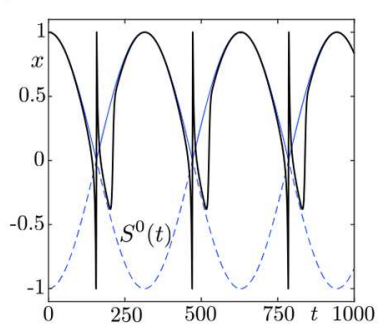

(a4)

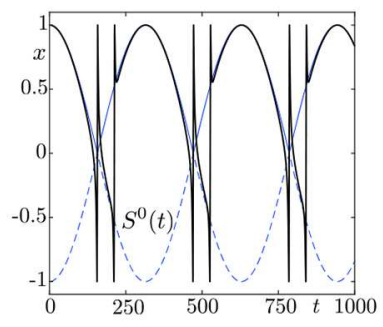

(b1)

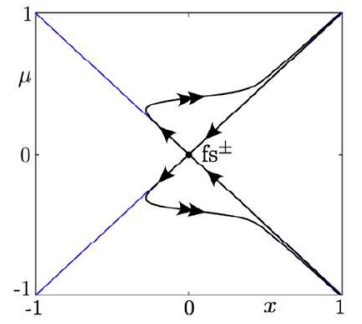

(b2)

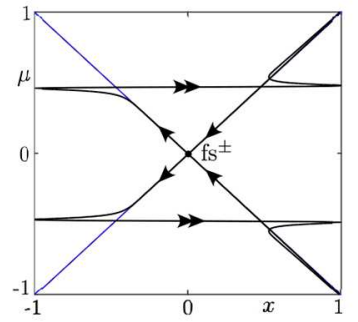

(b3)

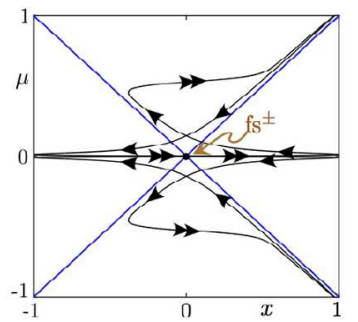

(b4)

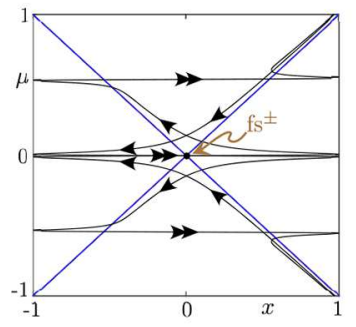

(c1)

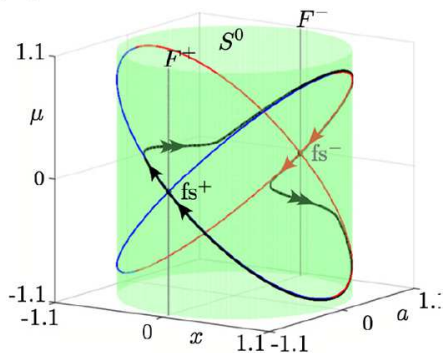

(c2)

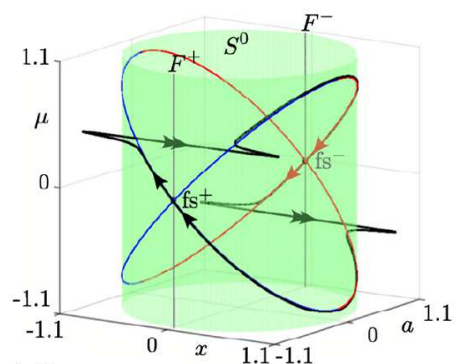

(c3)

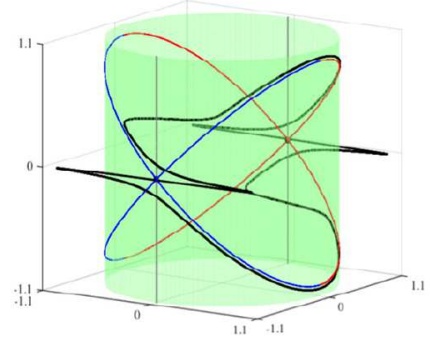

(c4)

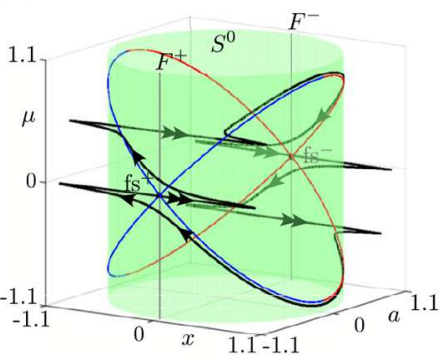

Figure 2. Spike-adding in system (2.4) for $a_{c}=\mu_{c}=0$. Panels (a1)-(a4) show the time profile in $x$ of all solutions together with the time-dependent critical manifold $S^{0}(t)$ (see Sect. 3 for definition); panels (b1)-(b4) display a planar projection of the solutions; panels (c1)-(c4) show phase-space representations of the solutions together with the critical manifold $S^{0}$ and its fold lines $F^{ \pm}$. In all panels, blue and red curves are two folded heteroclinic connections obtained as specific level sets of the Hamiltonian (2.14). All solutions contain canard segments: (a1)-(c1) spikeless headless canards; (a2)-(c2) two canards with head spikes; (a3)-(c3) two spikes and two headless canards, (a4)-(c4) two spikes and two canards with head spikes.

saddles $\mathrm{fs}^{ \pm}$. Additionally, right panels display the critical manifold $S^{0}$ and the fold lines $F^{ \pm}$. Finally, all left panels show the time profile for $x$ of these solutions together with the evaluation along $x(t)$ of the right-hand 
(a1)

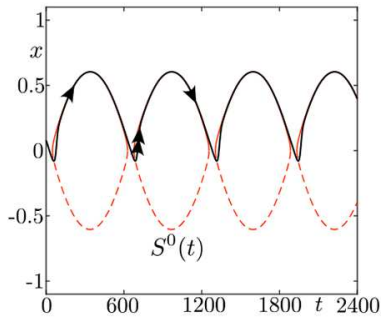

(a2)

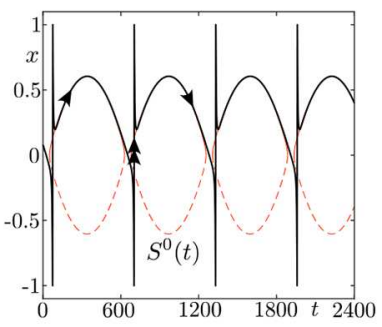

(b1)

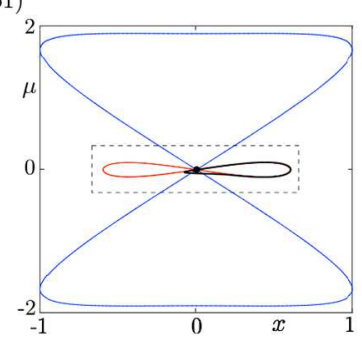

(b2)

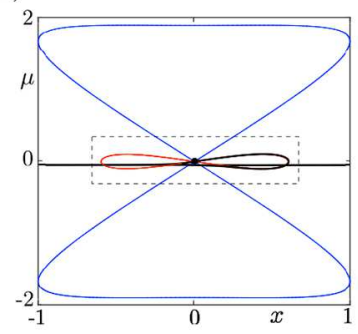

(c1)

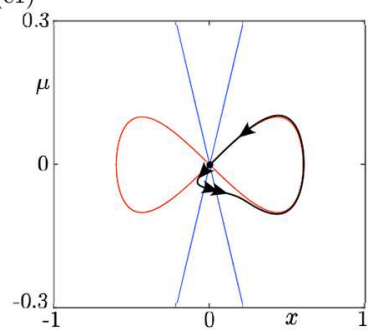

(c2)
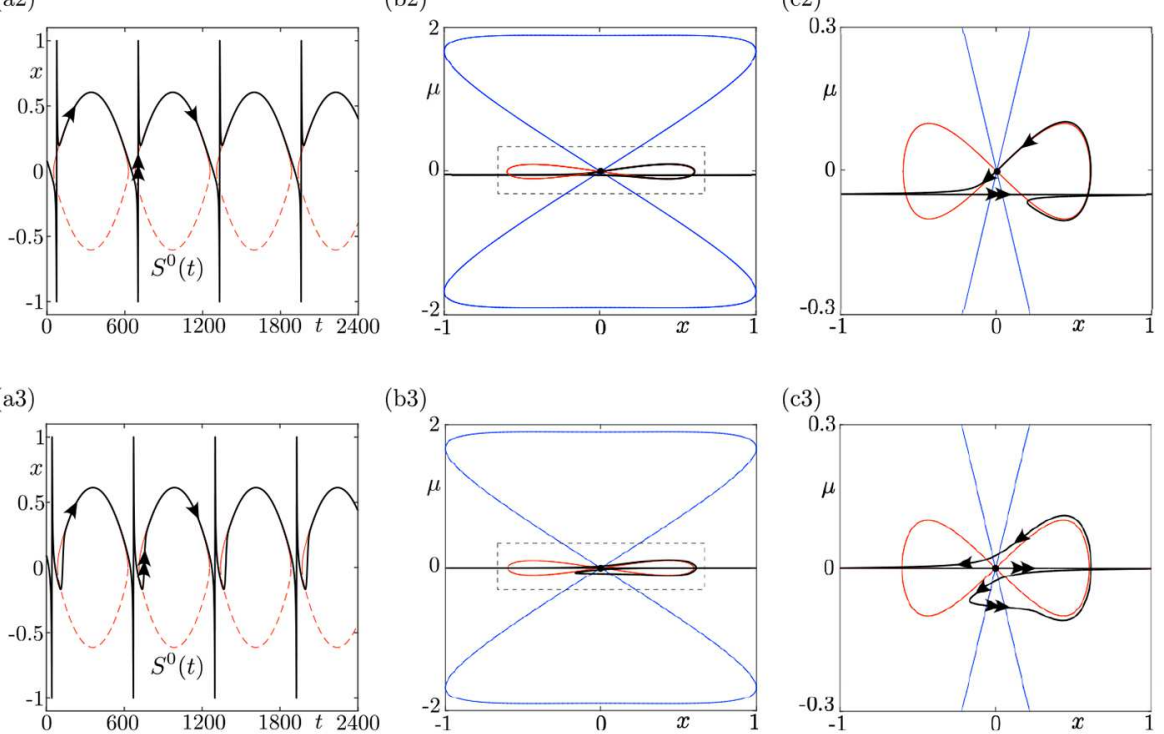

(b3)

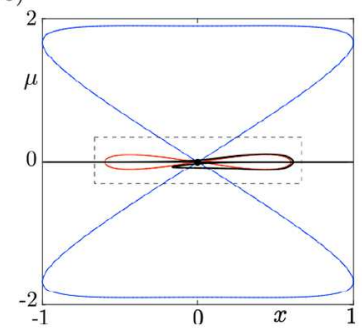

(c3)

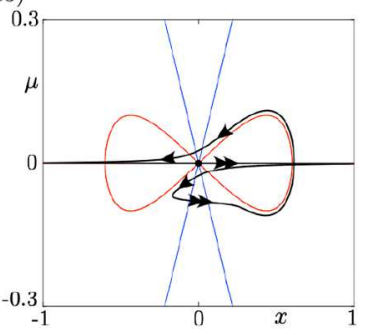

(c4)

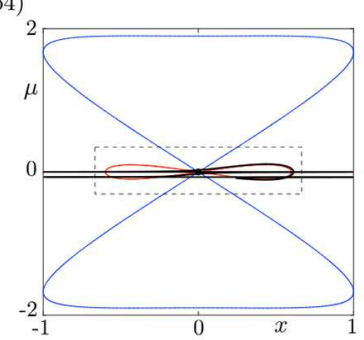

(d1)

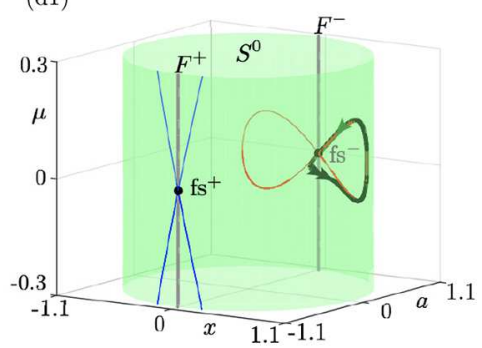

(d2)

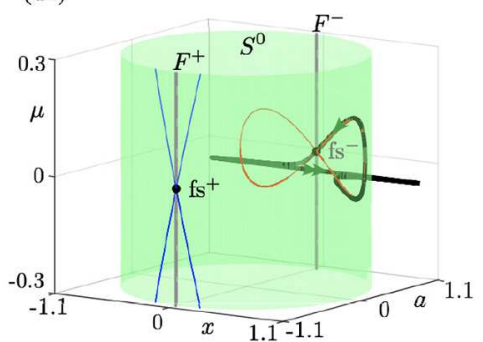

(d3)

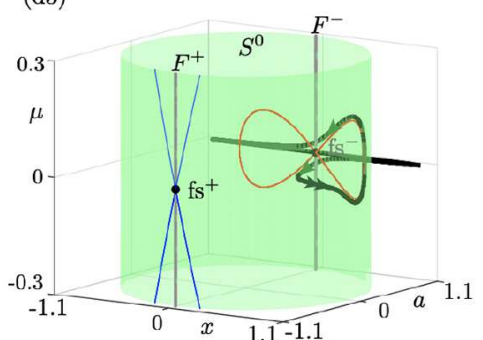

(d4)

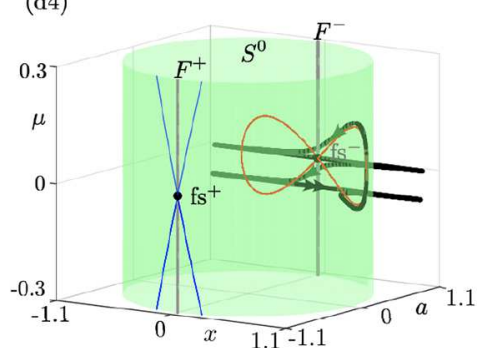

Figure 3. Spike-adding in system (2.4) for $\mu_{c}=0$ and $a_{c}=0.9$. Panels (a1)-(a4) show the time profile in $x$ of all solutions together with the time-dependent critical manifold $S^{0}(t)$ (see Sect. 3 for definition); panels (c1)-(c4) display zooms of panels (b1)-(b4); panels (d1)(d4) show phase-space representations of the solutions together with the critical manifold $S^{0}$ and its fold lines $F^{ \pm}$. In all panels, blue and red curves are folded homoclinic connections obtained as specific level sets of the Hamiltonian (2.14). All solutions contain canard segments: (a1)-(d1) spikeless headless canard; (a2)-(d2) canard with head spike; (a3)-(d3) one spike and one headless canard, (a4)-(d4) two spikes including one canards with head spike.

side of the differential equation for $x$, that is, the equation of the $x$-nullcline or critical manifold evaluation along $x(t)$; we will come back to this representation of the critical manifold, and its use to identify canard segments 
within time series, in Section 3. All solutions contain canard segments: (a1)-(b1) spikeless headless canards; (a2)-(b2) two canards with head spikes; (a3)-(b3) two spikes and two headless canards, (a4)-(b4) two spikes and two canards with head spikes.

When $a_{c} \neq 0$, this symmetry is broken and, depending on the sign of $a_{c}$, more and more spikes are added to the solutions near one of the two folded saddles: $\mathrm{fs}^{+}$(resp. fs ${ }^{-}$) for $a_{c}<0$ (resp. $a_{c}>0$ ). Figure 3 shows adding transitions near $\mathrm{fs}^{-}$for $a_{c}=0.9$. The two central panels show the $(x, \mu)$-plane, the latter ones corresponding to zoomed views of the former ones, while the right panels show the $(x, a, \mu)$-space, with the same dynamical objects as in Figure 2. The mechanism of spike-adding is similar to the symmetric case $a_{c}=0$ even though it only occurs close to one of the two folded saddles.

\subsection{Comparison with the classical example of biophysical parabolic burster: Plant model}

Our minimal system shares a number of similarities with Plant model, which is the main example of biophysical neuron model (i.e. based upon the Hodgkin-Huxley formalism) displaying parabolic bursting. Indeed, in [13] it was shown that Plant model, seen as a slow-fast dynamical system (even though there is no explicit small parameter) possesses a folded-saddle singularity and that spikes are added via transitions involving the canard (true and false) solutions associated with this folded saddle. System (2.4) possesses two folded saddles and the first added spikes, which corresponds to the transition from slow (spikeless) periodic solutions to solution with one or two (depending on the value of $a_{c}$ ) passes close to the folded saddles $\mathrm{fs}^{ \pm}$and follow their associated true canards. However, whether or not $a_{c}=0$, from the second spike-adding transition onward, spikes are added in the system via solutions that one can term jump-on canards [11] and they do not pass very close to folded saddles anymore. Indeed, as apparent in Figure 2a3-b3 and a4-b4, after the initial adding transitions, further spike are added from the other side (here, above) of the folded saddle and, hence, do not follow its true canard on the repelling side. Instead, trajectories involve in these spike-adding cross the fold line $F^{ \pm}$after having complete a fast segment and connect to a repelling slow manifold. This sequence attracting fast segment/repelling slow segment is what defines a jump-on canard. In Figure 2a3-b3 and a4-b4, the second (and subsequent) pair of spikes is added in this way. Furthermore, in the non-symmetric case corresponding to $a_{c} \neq 0$, a similar event occurs. In Figure 3a3-c3 and a4-c4, one clearly observes the second spike being added on the side of the folded saddle $\mathrm{fs}^{-}$but not so close to it, as the result of a jump-on canard explosion. Therefore, our minimal model display a spike-adding scenario that can be seen as a mix between the folded-saddle-canard adding scenario from Plant model, and the jump-on-canard scenario from the theta model from Ermentrout and Kopell; see [13] for details.

\section{LINK WITH THE DIPS AND SLICES BY J.E. LiTTLEWOOD}

Our minimal system's equation can be written as a one-dimensional ODE with a periodic forcing, namely

$$
\theta^{\prime}=a(\varepsilon t)-\sin \theta
$$

where $a$ is a circular function; for the numerical examples shown in Figure 4 , we will take $a(t)=K \sin t$. This comes from system (2.1) restricted to the periodic regime $r=1$ and with a slow oscillatory function for $a$ which we write as a time-dependent forcing instead of adding a harmonic oscillator as in equations (2.3). Posing $u=\tan \frac{\theta}{2}$, one can rewrite (after some rearranging) equation (3.1) as

$$
u^{\prime}=\frac{1}{2}\left(1+u^{2}\right) a(\varepsilon t)-u .
$$

While equation (3.2) is more amenable to analysis than equation (3.1), it is the opposite when it comes to numerical treatment; see our explanations below. These equivalent equations both take the form of a nonlinear ODE with a slow periodic forcing, a topic on which a large body of knowledge has been accumulated over the 

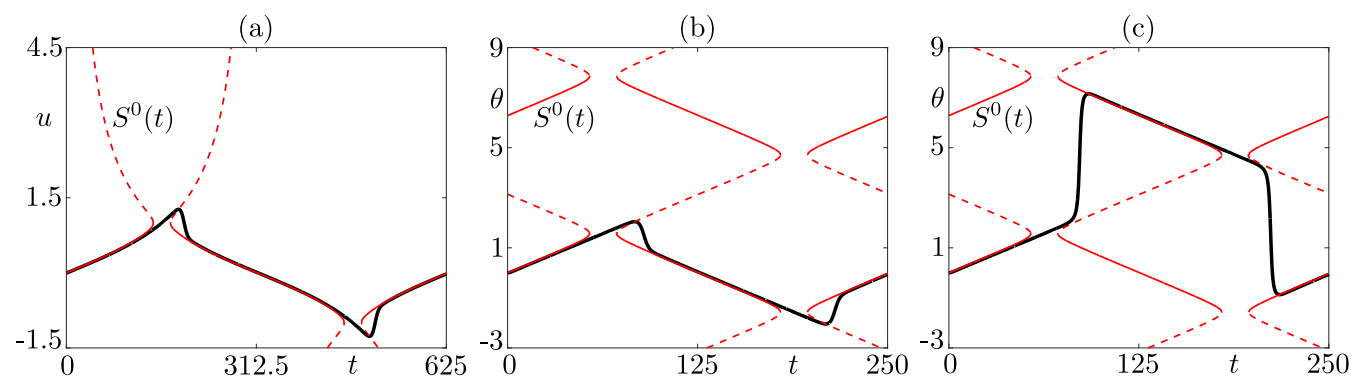

FiguRE 4. Time series of canard cycles of our minimal parabolic burster (3.1) with periodic forcing $a(\varepsilon t)$ for the chosen circular function $a(t)=K \sin t$; hence the forcing period is $T_{\varepsilon}=2 \pi / \varepsilon$. Also shown are the branches of the time-dependent critical manifold $S^{0}(t)$. The panels display the following informations: (a) headless canard for the model in $u$ (system (3.2)) with parameter values $K=1.01001$ and $\varepsilon=0.01$; (b) headless canard for the model in $\theta$ (system (3.1)) with parameter values $K=1.0253$ and $\varepsilon=0.025$; (c) canard with head for the model in $\theta$ with parameter values $K=1.0254$ and $\varepsilon=0.025$.

past few decades, that is, since a series of seminal papers by Cartwright and Littlewood on the forced van der Pol equation published over more than a decade from the mid-1940s until the late 1950s. Part III and IV of this series were published by Littlewood alone and in there he introduced the notions of dips and slices [32, 33]. These notions aimed to describe solutions of the forced van der Pol that follow an attracting motion up to a fold of the (now referred to as) critical manifold and then go past the fold line for a short segment after which either the solution goes back to the attracting region where it came from (this is the "dip") or it is pushed away towards the other attracting region, near the other fold line (this is the "slice"). Later on, Grasman further analysed these solutions $[20,21]$ and related dips and slices to canards in a recent entry on relaxation oscillations [22]. Canards in the singular limit $\varepsilon=0$ of the forced van der Pol system, in link with folded-saddle singularities, were studied by Szmolyan and Wechselberger in [46], and by Guckenheimer and collaborators in a series of paper [7, 23].

The idea of Littlewood is closely related to our minimal model is its form (3.2) and it can be summarised as follows. Considering (minimally) a one-dimensional ODE of the form

$$
x^{\prime}=f(x, a(\varepsilon t))
$$

where $a$ is a periodic function and $\varepsilon>0$ a small parameter. Then system (3.3) can be seen as a slow-fast system where $x$ is fast and $a$ is a periodic function of the slow variable $s=\varepsilon t$. In the formulation of (3.3), its critical manifold is time-dependent and periodic, it is given by the implicit equation $f(x(t), a(\varepsilon t))=0$. Similar to the case where one looks at these objects in phase space, one can identify parts of the time-dependent critical manifold that are attracting or repelling, simply by solving the additional implicit equation $f_{x}(x(t), a(\varepsilon t))=0$. Of course, one can rewrite the non-autonomous one-dimensional system with slow time-periodic forcing (3.3) as an autonomous two-dimensional slow-fast system of the form

$$
\begin{aligned}
x^{\prime} & =f(x, a(s)), \\
s^{\prime} & =\varepsilon,
\end{aligned}
$$

where the slow time-periodic forcing is now a term depending on the second state variable $s=\varepsilon t$ whose evolution equation is then simply a slow drift. In the phase plane of the slow-fast system (3.4), i.e. the $(s, x)$-plane, one typically plots trajectories of the solutions to the system together with its critical manifold $S^{0}=\{f(x, a(s))=0\}$. One can then observe the slow-fast transitions near non-normally hyperbolic point of $S^{0}$, in particular fold points, with respect to the fast variable $x$; again, the function $a$ being periodic in $s$, so is $S^{0}$. This is, for instance, 
what is done in Figure 1a where the phase space projection is three-dimensional and the critical manifold $S^{0}$ is a surface folded along a curve $F$ near which slow-fast transitions occur, namely transitions between the silent or quiescent phase and the active or burst phase of the bursting orbit. However, up to a rescaling by a factor $1 / \varepsilon$, one obtains the very same representation by plotting the time profile for $x$ of a solution of the original nonautonomous system (3.3) and superimpose on it the time-dependent critical manifold $S^{0}(t)$ implicitly defined as $\{f(x, a(\varepsilon t)=0\}$. Then, non-normally hyperbolic points still generically appear as either fold points with respect to $x$ or transcritical points; see the red curves in Figure 4. Furthermore, attracting (resp. repelling) parts of this time-dependent critical manifold satisfy $f_{x}(x, a(\varepsilon t))<0$ (resp. $f_{x}(x, a(\varepsilon t))>0$ ); they are represented by solid (resp. dashed) lines in Figure 4. In this way, one can easily identify canard segments in time series since they are located near repelling parts of the time-dependent critical manifold. Besides, starting from one-dimensional systems of the form (3.3) subject to a slow periodic forcing, this is the only way to identify canard solutions and it is entirely similar, in this context, to what is done in higher-dimensional slow-fast systems.

Following this approach, the time-dependent critical manifold of system (3.2) is shown in Figure 4a on top of a headless canard trajectory; the two canard segments are easily identified as the trajectory follows an attracting branch of $S^{0}(t)$ up to one of its fold points and then goes on to following a repelling branch of $S^{0}(t)$. These are headless canard segments since they are followed by a jump down in the direction of the fast variable $u$ towards a branch of $S^{0}(t)$ with similar values of $u$ as before the canard segment. In the language of Littlewood, this corresponds to a dip. Figure $4 \mathrm{~b}$ and $\mathrm{c}$ shows similar objects for the $\theta$-version of this system, defined by equation (3.1): the plotted solution is a headless canard in (b) and a canard with head in (c). Indeed, in the former case, the solution jumps down after the canard segment towards a branch of $S^{0}(t)$ with similar values of $\theta$ as before the canard segment, whereas in the latter case the solution jumps up towards a branch of $S^{0}(t)$ with values of $\theta$ that are higher by an amount of approximately $\pi$ than the values of the branch of $S^{0}(t)$ followed by the solution before its canard segment. In Littlewood's terms, the solution shown in (b) displays a dip (headless canard segment) while the one shown in (c) displays a slice (canard segment with head). Note that, in the $u$ coordinate, the system cannot exhibit canards with head as these correspond to a jump up in $\theta$ of approximately $\pi$, which means that $u$ diverges to $+\infty$. Indeed, in the $u$-variable, the time-dependent critical manifold has only one stable segment, which means that only dips (headless canards) are possible; see Figure 4a. This is in contrast with what happens in the $\theta$-variable, where the time-dependent critical manifold has multiple stable segments, hence allowing for both dips (headless canards) and slices (canards with head).

Let us now summarise the comparison between the slow-fast analysis of system (3.3) in the time domain using the time-dependent critical manifold $S^{0}(t)$, and the more classical slow-fast analysis of system (3.4) in the phase plane using the usual critical manifold $S^{0}$. The classical approach corresponds to performing what is typically referred to as slow-fast dissection [39] and $S^{0}$ corresponds to the bifurcation diagram for equilibria of the fast subsystem associated with system (3.4), which effectively is the same equation as (3.3) but with a bifurcation parameter $s$ as opposed to the slow time-dependent term $\varepsilon t$. Hence, obtaining the equilibrium bifurcation diagram of this fast subsystem in $s$ (by direct manipulation of the equations or by means of numerical bifurcation analysis) and then plotting it superimposed on top of a periodic solution of the full system (3.4) exactly amounts to performing a slow-fast dissection. Instead, plotting the time-dependent critical manifold of system (3.3) in time on top of that periodic solution is effectively plotting a rescaled version of the bifurcation diagram of the fast subsystem of (3.4), with rescaling factor being $1 / \varepsilon$. Thus, the two approaches are very similar and provide similar type of information. The latter one is simply adapted to system of the form of (3.3), which are sometimes referred to as adiabatic systems [4]; on this topic, we refer the reader to the work of Berglund $[3,5]$ in which time-dependent critical manifolds are superimposed in the time domain onto periodic solutions of such systems, yet without references to Littlewood's dips and slices and no direct link made with canards. Without directly referring to the work of Littlewood, Marc Diener has studied similar representation of canards in one-dimensional dynamical systems with slow periodic forcing $[15,16]$. Finally, the idea was also present in the doctoral work of Callot [8].

We remark that this approach to characterise different canard solutions in the time domain also works in planar slow-fast systems with a constant forcing. As before, it requires to be able to solve (at least numerically) the implicit equation corresponding to the critical manifold for the fast variable. The prototypical autonomous 
(a)

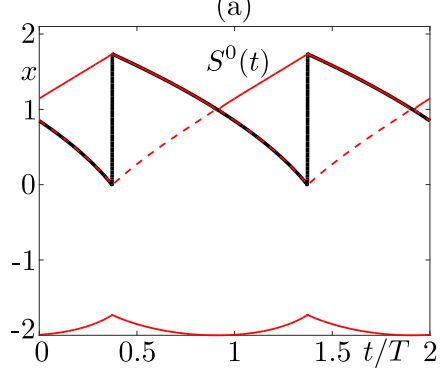

(b)

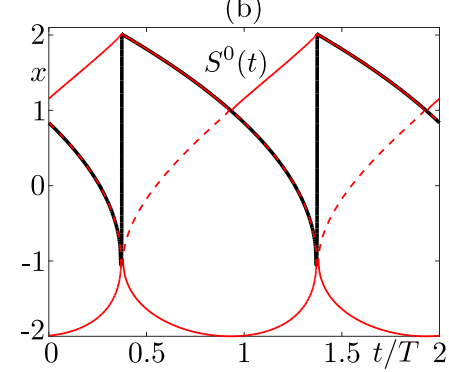

(c)

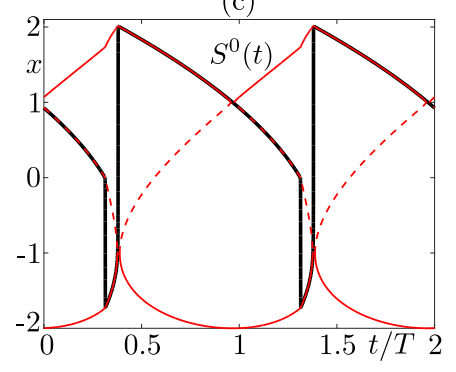

FiguRE 5. Time series of canard cycles of the van der Pol oscillator (3.5) together with the three branches of the critical manifold (obtained after the solving the equation of the $x$-nullcline for $x$ ) evaluated along each cycle, for $\varepsilon=0.001$ and $a \approx 0.99987490608$ (the difference in $a$ across the canard explosion goes beyond the 11th decimal place). (a) Headless canard (dip); (b) maximal canard (transition dip/slice); (c) canard with head (slice).

canard system in the plane is the van der Pol oscillator with constant forcing, in which canards where first identified and analysed as such [2]. The system's equations read

$$
\begin{aligned}
& x^{\prime}=y-x^{3} / 3+x, \\
& y^{\prime}=\varepsilon(a-x) .
\end{aligned}
$$

Since the critical manifold is the graph of a cubic polynomial in the fast variable $x$, one can solve it for $x$ by radicals using Cardano's formula. In this way, one can plot the time-dependent critical manifold $S^{0}(t)$ for this autonomous system. In Figure 5, we show the result of this procedure for a headless canard (a), the maximal canard (b) and a canard with head (c). The same phenomenon as in the forced van der Pol occurs, and it confirms that, in the most classical and historical sense, headless canards correspond to dips while canards with head correspond to slices. At the transition between dips and slices, the boundary between these two sub-families of canards, namely the maximal canard, corresponds to the longest dip possible.

\section{Conclusion}

This article introduces a minimal model of parabolic bursting with integrable slow flow, obtained by forcing harmonically a minimal model of an SNIC oscillator. We analyse this new parabolic burster, in particular its slow flow and spike-adding structure. We further discuss the links with a classical work of J.E. Littlewood. We believe that this approach can help further understanding the parabolic bursting's main properties in biophysical models, through minimal models. An important question in neuroscience is that of encoding of information (coming from external stimuli) via neuronal activity. There are several paradigms underpinning this question and associated hypotheses regarding how the neural coding is implemented by the brain. Rate coding considers that the rate of neural activity (i.e. the firing rate) is the main quantity that encodes information; this is the basic of neural networks. Other approaches consider that spikes and their timing are equally (if not more) important, and this has led to the emergence of spiking neural networks. However, spikes are very rapid events and there is a third approach which considers that groups of spikes or bursts have a stronger chance to possess an encoding power. In the context of burst coding [27,31], it is therefore important to be able to control the size of bursts in given neuron models. Hence, spike-adding transitions become potential key regulator of neural activity and of neural coding. We believe that minimal models as the one proposed here are important as they offer further amenities to analysis and control while being computationally tractable which, once embedded in a network architecture, could bring new insight into this coding through bursts. This is clearly beyond the scope of the present paper and will be a very interesting topic of future work. 


\section{REFERENCES}

[1] S.M. Baer, J. Rinzel and H. Carillo, Analysis of an autonomous phase model for neuronal parabolic bursting. J. Math. Biol. 33 (1995) 309-333.

[2] É. Benoît, J.-L. Callot, F. Diener and M. Diener, Chasse au canard. Collect. Math. 32 (1981) 37-119.

[3] N. Berglund and H. Kunz, Chaotic hysteresis in an adiabatically oscillating double well. Phys. Rev. Lett. 78 (1997) $1691-1694$.

[4] N. Berglund, Adiabatic dynamical systems and hysteresis. Ph.D. thesis, Department of Physics, École Polytechnique Fédérale de Lausanne (EPFL), no. 1800 (1998). Available at: https://infoscience.epfl.ch/record/32277/files/EPFL_TH1800.pdf

[5] N. Berglund and H. Kunz, Memory effects and scaling laws in slowly driven systems. J. Phys. A: Math. Gen. 32 (1999) 15-39.

[6] R. Bertram, M.J. Butte, T. Kiemel and A. Sherman, Topological and phenomenological classification of bursting oscillations. Bull. Math. Biol. 57 (1995) 413-439.

[7] K. Bold, C. Edwards, J. Guckenheimer, S. Guharay, K. Hoffman, J. Hubbard, R. Oliva and W. Weckesser, The forced van der Pol equation II: Canards in the reduced system. SIAM J. Appl. Dyn. Syst. 2 (2003) 570-608.

[8] J.-L. Callot, Bifurcations du portrait de phase pour des équations différentielles linéaires du second ordre ayant pour type l'équation d'Hermite. Ph.D thesis, Université de Strasbourg, France (1981).

[9] F. Clément and J.-P. Françoise, Mathematical modeling of the GnRH pulse and surge generator. SIAM J. Appl. Dyn. Syst. 6 (2007) 441-456.

[10] C.A. Del Negro, C.F. Hsiao, S.H. Chandler and A. Garfinkel, Evidence for a novel bursting mechanism in rodent trigeminal neurons. Biophys. J. 75 (1998) 174-182.

[11] P. De Maesschalck, F. Dumortier and R. Roussarie, Canard-cycle transition at a fast-fast passage through a jump point. C. R. Math. 352 (2014) 27-30.

[12] M. Desroches, T.J. Kaper and M. Krupa, Mixed-mode bursting oscillations: dynamics created by a slow passage through spike-adding canard explosion in a square-wave burster. Chaos 23 (2013) 046106.

[13] M. Desroches, M. Krupa and S. Rodrigues, Spike-adding in parabolic bursters: the role of folded-saddle canards. Phys. D 331 (2016) 58-70.

[14] M. Desroches and V. Kirk, Spike-adding in a canonical three-time-scale model: superslow explosion and folded-saddle canards. SIAM J. Appl. Dyn. Syst. 17 (2018) 1989-2017.

[15] M. Diener, Nessie et les canards, Institut de Recherche Mathématique Avancée. Preprint IRMA-76-P-38 (1979).

[16] M. Diener, Deux nouveaux "phénomènes-canard". C. R. Acad. Sci. Paris Ser. A 290 (1980) 541-544.

[17] F. Dumortier and R. Roussarie, Canard cycles and center manifolds, Memoirs of the American Mathematical Society, Rhode Island (1996) 577.

[18] G.B. Ermentrout and N.J. Kopell, Parabolic bursting in an excitable system coupled with a slow oscillation. SIAM J. Appl. Math. 46 (1986) 233-253.

[19] M. Golubitsky, K. Jǒsić and T.J. Kaper, An unfolding theory approach to bursting in fast-slow systems, in Global Analysis of Dynamical Systems, edited by H.W. Broer, B. Krauskopf and G. Vegter, CRC Press, Boca Rotan, US (2001) 282-313.

[20] J. Grasman, H. Nijmeijer and E.J.M. Veling Singular perturbations and a mapping on an interval for the forced van der Pol relaxation oscillator. Phys. D 13 (1984) 195-210.

[21] J. Grasman, Asymptotic Methods for Relaxation Oscillations and Applications. Springer, Berlin (1987).

[22] J. Grasman, Relaxation oscillations, in Encyclopedia of Complexity and Systems Science, edited by R.A. Meyers, Springer, New York, (2009) 7602-7616.

[23] J. Guckenheimer, K. Hoffman and W. Weckesser, The forced van der Pol equation I: the slow flow and its bifurcations. SIAM J. Appl. Dyn. Syst. 2 (2003) 1-35.

[24] J. Guckenheimer and Y. Ilyashenko, The duck and the devil: canards on the staircase. Moscow Math. J. 1 (2001) $27-47$.

[25] A.L. Hodgkin and A.F. Huxley, A quantitative description of membrane current and its application to conduction and excitation in nerve. J. Physiol. 117 (1952) 500-544.

[26] E.M. Izhikevich, Neural Excitability, Spiking, and Bursting. Int. J. Bifurc. Chaos 10 (2000) 1171-1266.

[27] E.M. Izhikevich, N.S. Desai, E.C. Walcott and F.C. Hoppensteadt, Bursts as a unit of neural information: selective communication via resonance. Trends Neurosci. 26 (2003) 161-167.

[28] E.M. Izhikevich, Dynamical Systems in Neuroscience: The Geometry of Excitability and Bursting, MIT press. Cambridge (2007).

[29] A. Kepecs and X.J. Wang, Analysis of complex bursting in cortical pyramidal neuron models. Neurocomputing 32 (2000) 181-187.

[30] M. Krupa and P. Szmolyan, Relaxation oscillation and canard explosion. J. Differ. Equ. 174 (2001) $312-368$.

[31] J.E. Lisman, Bursts as a unit of neural information: making unreliable synapses reliable. Trends Neurosci. 20 (1997) 38-43.

[32] J.E. Littlewood, On non-linear differential equations of the second order: III. The equation $\ddot{y}-k\left(1-y^{2}\right) \dot{y}+y=b \mu k \cos (\mu t+\alpha)$ for large $k$, and its generalizations. Acta Math. 97 (1957) 267-308.

[33] J.E. Littlewood, On non-linear differential equations of the second order: IV. The general equation $\ddot{y}+k f(y) \dot{y}+g(y)=$ $b k p(\varphi), \varphi=t+\alpha$. Acta Math. 98 (1957) 1-110.

[34] S. Moran, S.M. Moenter and A. Khadra, A unified model for two modes of bursting in GnRH neurons. J. Comput. Neurosci. 40 (2016) 297-315.

[35] R.E. Plant and M. Kim, On the mechanism underlying bursting in the Aplysia abdominal ganglion R15 cell. Math. Biosci. 26 (1975) 357-375. 
[36] R.E. Plant and M. Kim, Mathematical description of a bursting pacemaker neuron by a modification of the Hodgkin-Huxley equations. Biophys. J. 16 (1976) 227-244.

[37] R.E. Plant, The effects of calcium ${ }^{++}$on bursting neurons: a modelling study. Biophys. J. 21 (1978) $217-237$.

[38] R.E. Plant, Bifurcation and resonance in a model for bursting nerve cells. J. Math. Biol. 11 (1981) $15-32$.

[39] J. Rinzel, A formal classification of bursting mechanisms in excitable systems, in Proc. of the International Congress of Mathematicians. Berkeley, California (1986) 1578-1593.

[40] J. Rinzel and Y.S. Lee, Dissection of a model of neuronal parabolic bursting. J. Math. Biol. 25 (1987) $653-675$.

[41] I.V. Schurov, Ducks on the torus: existence and uniqueness. J. Dyn. Control Syst. 16 (2010) 267-300.

[42] I.V. Schurov, Canard cycles in generic fast-slow systems on the torus. Trans. Moscow Math. Soc. 71 (2010) 175-207.

[43] I.V. Schurov and N. Solodovnikov, Duck factory on the two-torus: multiple canard cycles without geometric constraints. J. Dyn. Control Syst. 23 (2017) 481-498.

[44] P. Smolen, D. Terman and J. Rinzel, Properties of a bursting model with two slow inhibitory variables. SIAM J. Appl. Math. 53 (1993) 861-892.

[45] C. Soto-Treviño, N. Kopell and D. Watson, Parabolic bursting revisited. J. Math. Biol. 35 (1996) 114-128.

[46] P. Szmolyan and M. Wechselberger, Canards in $\mathbb{R}^{3}$. J. Differ. Equ. 177 (2001) 419-453.

[47] D. Terman Chaotic spikes arising from a model of bursting in excitable membranes. SIAM J. Appl. Math. 51 (1991) 1418-1450. 